\title{
A Developed Sensor for Color Identification, Sorting and Counting Automation Control System
}

\author{
Hong Yu, Alex Pyankov, and Marcus Perla
}

\begin{abstract}
Knowing how to manipulate the data from environment to automation control systems in industry can innovate and improve a quality of the products effectively. In plastic manufacturing, there is a need of automated control system that can recognize color and sort, count and sequentially control processing since the certain quantity of color billets mix into a batch in order to produce desired colorful products. In this paper, a color recognition sensor based on the principle of LEDs energy harvest corresponding actuator has been designed. The derivational voltage due to red, green and blue color shift in color recognition, sorting and counting automation control system is measured as a signal input of the controller that works with the reflected light properties such as the reflective harmonic energy. Sequentially, the automation control system with human machine interface (HMI) and supervisory control and data acquisition (SCADA) will monitor the quantity of diversity color products in intuitive and remote supervisory, customization or adjustments in automation processing. Furthermore, the designed device will integrate into artificial intelligent (AI) and unmanned aerial vehicle (UAV).
\end{abstract}

Index Terms - color identification, sorting, counting, automation control system.

\section{INTRODUCTION}

In recent years, the sensors have been popularly used everywhere such as Artificial Intelligent (AI), medical healthcare and industry, especially color sensors in plastic manufacture. Typically, the detected raw photocurrents are extremely low amplitude and invariably require amplification to convert the photocurrents to useable levels. The most practical analog-output color sensors incorporate a transmission impedance amplifier and provide voltage outputs. Generally, light falling upon each of the photodiodes converts to a proportional photocurrent. a silicon photodiode responds to wavelengths ranging of the ultraviolet region through the visible, with a peak response region of between 800 and $950 \mathrm{~nm}$. The red $(580 \mathrm{~nm})$, green $(540 \mathrm{~nm})$ and blue $(450 \mathrm{~nm})$ transmissivity color filters reshape and optimize the photodiode's spectral response that dovetails with the requirements [1].

There are two color-sensing modes: reflective and transmission [1]. In reflective sensing, the color sensor detects light reflected from a surface or object, with both the light

Submitted on January 31, 2021

Published on February 15, 2021.

Hong Yu, Engineering Technology Department, Fitchburg State University, IEEE senior membership, USA.

(e-mail: yhong2006@gmail.com) source and the color sensor placed close to the target surface. For example, white light incident onto a red surface is reflected as red. The reflected red light impinges on the color sensor producing Red, Green and Blue output voltages. By interpreting the three voltages, the color can be determined. Since the three output voltages increase linearly with the intensity of the reflected light, the color sensor also measures the reflectivity of the surface or object. In the transmission mode of operation, the sensor faces the light source. The filter-coated photodiode array of the color sensor converts the incident light into red, green and blue (RGB) photocurrents that converted to analog voltages. Since all three outputs increase linearly with increasing light intensity, the sensor can measure both the color and total intensity of the light.

Typically, Photovoltaic cells convert incoming photons into electricity. Efficiencies range from 5\% to $30 \%$, depending on the material used. Indoor the illumination levels are much lower $\left(10-100 \mathrm{lW} / \mathrm{cm}^{2}\right)$ and photovoltaic cells generate a surface power density similar or slightly larger than that of the harvesters described above. Indoor use requires a fine-tuning of the cell design to the different spectral composition of the LED light and the lower level of illumination [3].

The photovoltaic effect of the LED array uses to harvest energy with the device. Harvested energy is stored in circuit for powering LED array. The powering LED energy harvester for various commercially available LEDs will determine the most suitable LED type with respect voltage gain and current drivability. The power consumption of presented LED is optimized by using response surface methodology (RSM) with respect to the following input parameters: frequency of operation, frequency of data transmission, the data with respect to the consumed power and the reflection or transmission distance. The optimization proposes optimal input parameters for minimum power consumption and operating distance that presents a compromise between the aforementioned parameters in reference to the input variables [2]. The following Fig. 1 shows an energy harvested voltage vs current. Fig. 2 shows the measured spectral responses corresponds the measured spectral responses.

Response surface methodology (RSM) is a set of techniques from mathematics and statistics used for empirical model building and model optimization. It is mostly used for

Alex Pyankov, Engineering Technology Department, Fitchburg State University, IEEE student membership USA.

Marcus Perla, Engineering Technology Department, Fitchburg State University, IEEE student membership, USA. 
process modelling and optimization for minimum power in the processing. Related work on LEDs energy harvest or power consumption circuit have been optimized.

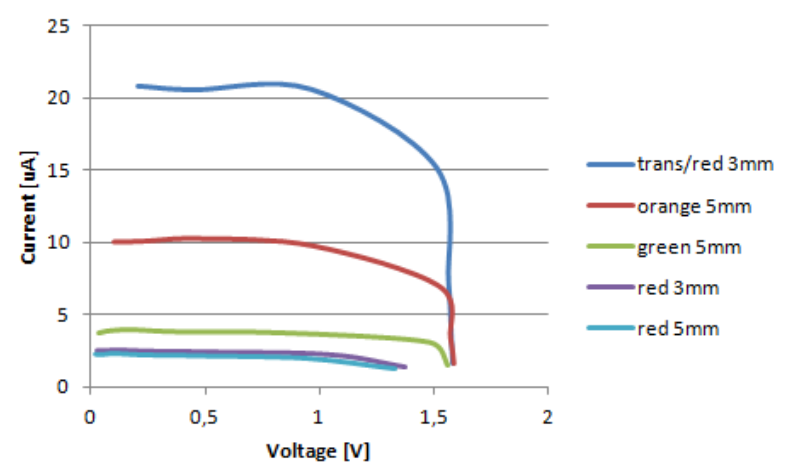

Fig. 1. Voltage vs. current.

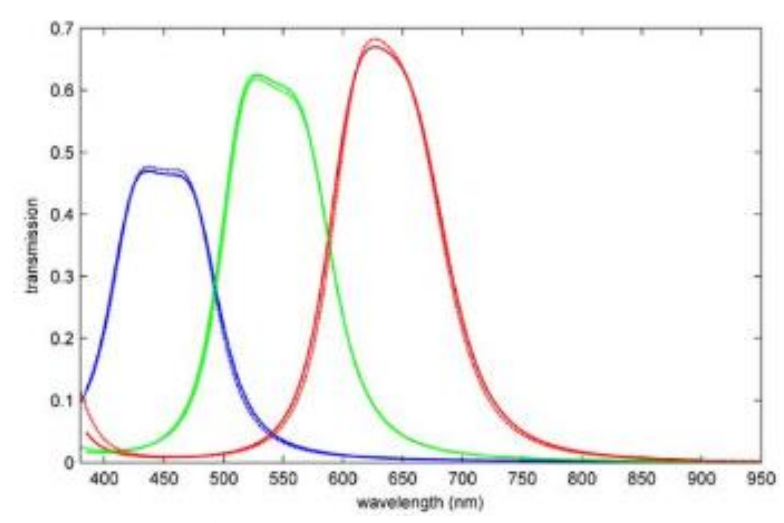

Fig. 2. measured spectral responses.

The output of an energy harvest is not directly suited as the power supply for circuits because of variable voltage over time and a voltage or current management circuit is required.

The voltage unit should handle the very low feeding power and be able to adapt its input to the energy harvester and its output to the load [3]. Actually, the energy harvester will be measured and evaluated by human machine interface (HMI) and supervisory control and data acquisition (SCADA). The variable treated as the input parameters whereas the model response parameter is power consumption represented as voltage. Fig. 2 shows the measured spectral responses of RGB filters [4].

In the past years, the plastic manufacturing has required certain quantity of certain color billets to be mixed in a batch in order to produce desired parts color. Therefore, there is a need for a system that can automatically recognize RGB colors and sequentially control manufacturing process, meanwhile calculating a weight, cost and quantity of a product. In this paper, a circuit with sensor to recognize, sort and count RGB color has been designed and integrated to automation control system. The system involves modern sophisticated ways of automation control and monitoring such as HMI and SCADA. As an essential part of HMI, Programmable Logic Controller (PLC) is the interface device used for communicating to the designed circuit and collect the data reliability in modern industry. On the other hand, the system should allow to initial setup an intuitive and easy way of customization or adjustment as a need for friendly interface HMI.

\section{Color Recognition Device Circuit}

Color recognition device circuit is designed on a principle of the reflected color light. Each color has different reflection properties such as darker color reflects less and absorb more light energy. This quality can be used for color recognition by measuring reflected light energy. The basic functional concept of the LEDs energy harvesting and red, green, or blue reflective sensing, a color recognition, sorting and counting automation control system has been designed. The following Fig. 3 shows the flow chat of the control system.

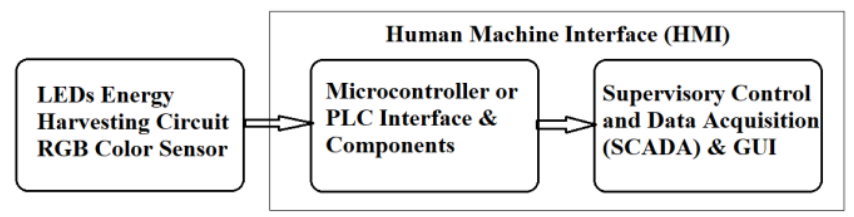

Fig. 3. Automation Control System Flow Chart.

In this section, the main characteristics of the designed energy harvesting circuit will enhance the reflective energy sensing. The circuit consists of two parallel-connected LEDs and detects the different color reflecting energy consumption. If narrowband light sources are optimized for color object reflectance while preserving color appearance, there is a large variance in absorption values due to the difference in the spectral reflectance characteristics [5]. Since the optimized spectra resulted in reduced energy consumption and absorption, it reduced the energy consumption in the circuit. Therefore, it is also important to note that this study investigates absorption-minimizing spectra for single colored objects.

Since red, green, blue colors have the different reflection properties, Absorption minimization and effective energy approaches can be utilized to quantify in voltages and the quality energy can be used to design color recognition device. Fig. 4 shows the designed color recognition device and Fig. 5 shows the designed sensor circuit.

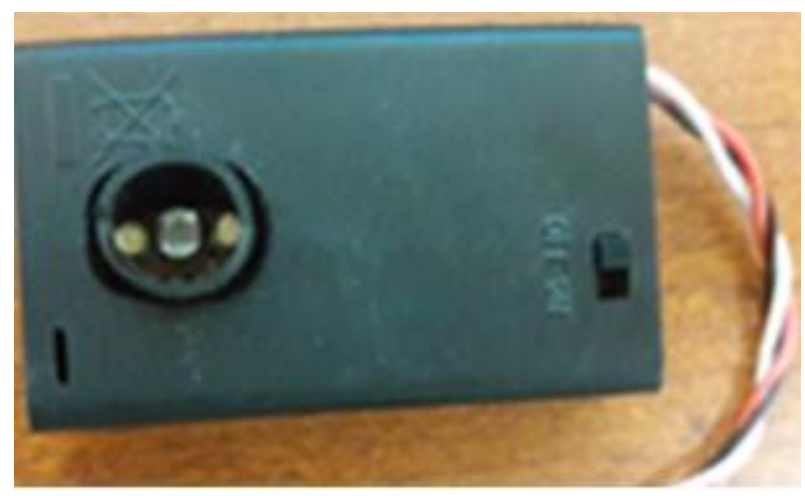

Fig. 4. The designed color recognition sensor device. 


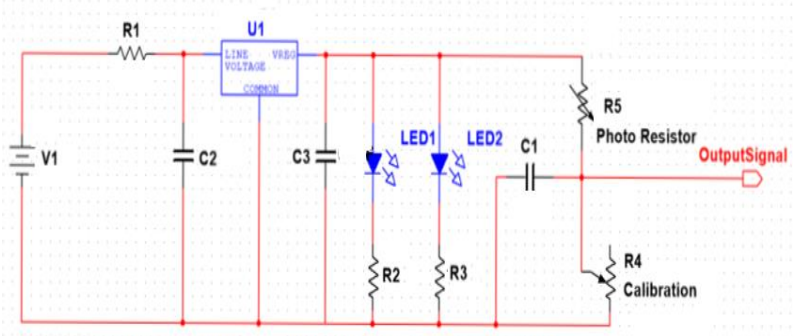

Power Supply 5...30 VDC VS Output Power range 0...5 VDC

Fig. 5. The designed circuit of the color sensor device.

In order to measure reflected light energy, a steady light source is needed. Two LEDs LED1 and LED2 are chosen as a light source. A photo resistor R5 is a light sensitive resistor that changes its resistance depending on the light. The voltage divider circuit is used with the photo resistor R5 in series with a calibration potentiometer $\mathrm{R} 4$. The nominal resistance of the photo resistor matches nominal resistance of potentiometer. A microcontroller or a PLC can easily read the output voltage from this sensor. In this case, PLC has an analog input expansion card with analog-to-digital converter (ADC) that allows interpreting voltage to a digital data.

The sensor design was chosen to have an integrated voltage regulator $\mathrm{U} 1$ with input $\mathrm{C} 2$ and output $\mathrm{C} 3$ capacitors. Resistor $\mathrm{R} 1$ is assisting with heat dissipation of the voltage regulator $\mathrm{U} 1$ by slightly dropping the input voltage since the PLC supplied input voltage $\mathrm{V} 1=24 \mathrm{~V}$ is nearly at the max of the voltage regulator input voltage. $\mathrm{C} 1$ capacitor is assisting with voltage spikes in the voltage divider circuit. R2 and R3 are current limiting resistors since LEDs have virtually no internal resistance and will be short circuit otherwise. Since the ambient light is different depending on the location which can affect a default or the nominal resistance of the photo resistor. Calibration is not always necessary but can be done by adjusting the potentiometer, which balances two resistance values R5 and R4 in the voltage divider circuit.

\section{SCADA AND HMI SYSTEM}

In this paper, HMI includes PLC and SCADA as hardware and software. SCADA design a touchscreen panel assisting in the monitoring and control of manufacturing processes. HMI usually works with other manufacturing facilities. SCADA is a software that overlays PLC operation, sorts and counts at a remote control station. Fig. 6 shows color recognition device with the HMI automation control system.

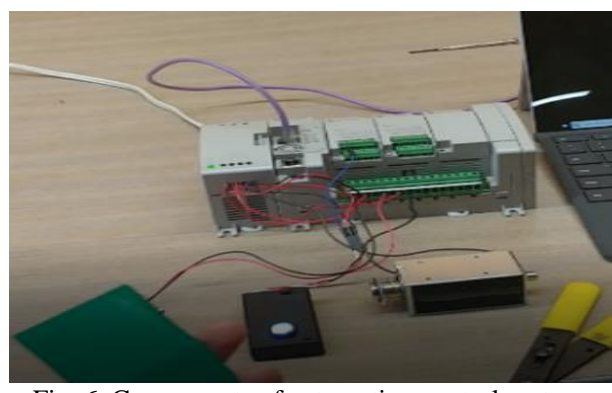

Fig. 6. Components of automation control system.

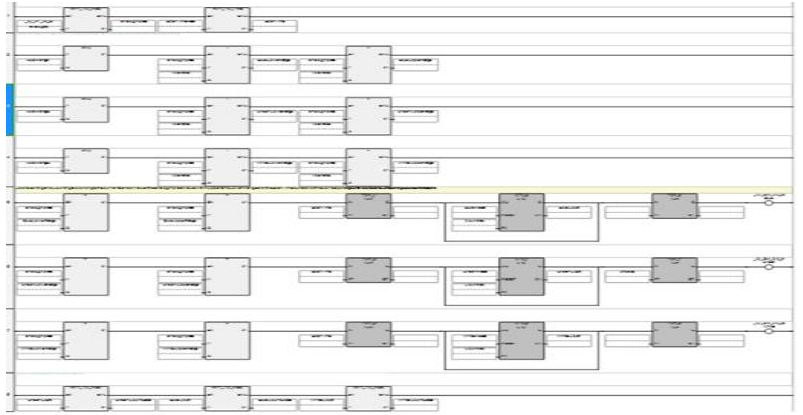

Fig. 7. The partial codes of PLC.

Generally, PLCs are designed to control complex industrial processes such as running machines and motors. In this HMI, it is designed to measure the output voltage of the designed device. It is a simple programmable and fully scalable to an operation's requirements. It is capable of performing much more complex tasks. Data from the PLC are relayed to the system and commands are entered into the HMI to adjust the processes they control. Fig. 7 shows the partial code of PLC of HMI.

SCADA is a central system used to monitor and run plant processes. SCADA is used in conjunction with other devices. It is typically software installed on a computer and one of its major functions is to act as an interface with industrial machines or other HMI. In other words, it allows users to track information coming from equipment, enter commands and make changes to their programming. In this section research, PLC will measure the power consumption in the energy of color recognition action is the process of energy changes in the designed device, which can only be initiated by radiation. Output from RGB designed device is connected to HMI system such as an analog input of PLC. The power consumption as the input voltage from the designed device converted by the analog digital converter (ADC) into a digital value. This digital value is compared to a set value range. Each color has its value range. The value range is set by putting a color part on the RGB sensor device pressing the remember button on the SCADA screen of the HMI. PLC remembers this specific value and by adding and subtracting the range. It will have a specific color value range. Range or sensitivity can be adjusted from the SCADA screen. Three channels to recognize, sort and count for the LEDs energy harvest corresponding actuator will communicate with SCADA. This system has a scan time adjustment, scan time is the duration of the output within value range in order to be counted. Fig. 8 shows the designed screen by SCADA.

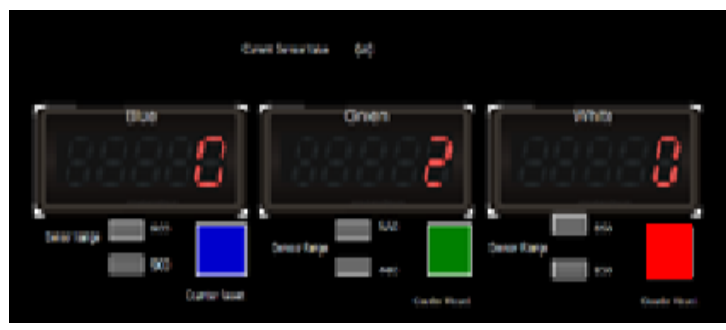

Fig. 8. The designed screen by SCADA. 
In HMI system, SCADA communicates with PLC via Ethernet protocol accessing an IP address remotely from anywhere there is an internet access. The designed interface instantly displays the collected data, sorts and counts the color, weight of material by SCADA at remote workstation. Fig. 9 shows the circumstace of HMI.

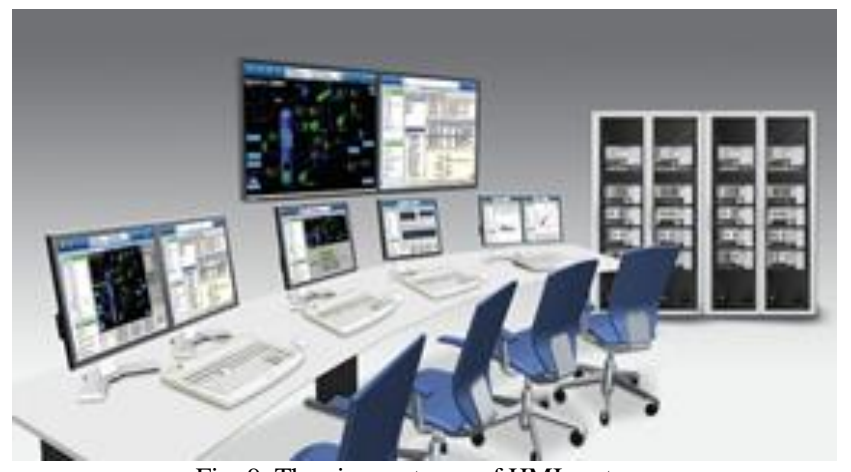

Fig. 9. The circumstance of HMI system.

\section{EXPERIMENTAL Methodology AND ANALYSIS}

In the designed device with the LEDs light circuit, the response surface methodology (RSM) from mathematics and statistics is used for model building and model optimization. It is mostly used for process modelling and optimization for minimum power in the processing. Power consumption has a significant impact on the circuit performances and on the amount of energy. In order to validate the power consumption of RGB surface response, the measurements of LED energy harvest are performed. When two LEDs setup as the light sources, it will use to enhance the color appearance of RGB objects. The distance between two LEDs is $1 / 2$ inch. An IR sensor have been sited at the center of two LEDs. These narrowband light emitting diodes (LEDs) mix to rejuvenate the color appearance in the designed device circuit. Fig. 10 shows the narrowband reflective sensing. Fig. 11 shows the reflected light and spectral strength.
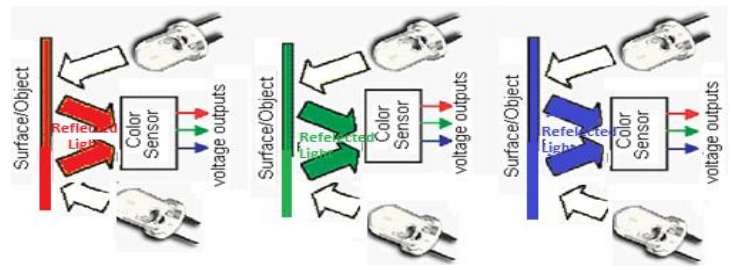

Fig. 10. The narrowband reflective sensing.

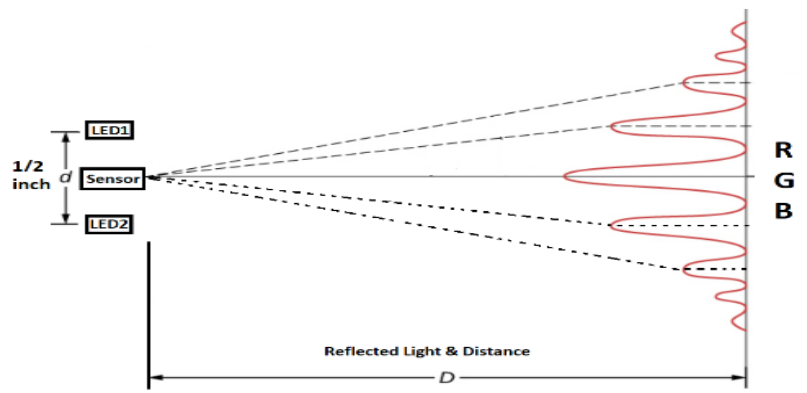

Fig. 1.1 The reflected light and distance.
On the other hand, the topological differences of a defecttype due to size or depth do not prohibit extraction of specific elements. An effective interrelation may cause on the surface to allow its visualization. This interrelation it is narrowing down the role of defect's width or size and its position on the surface. Further, a small defect near the surface is by analogy as effective as a large defect far from the surface [7]. The reflected light also depends on the appearance chrome. It assesses to prevent the reduction in the color quality of an item after the energy harvest. IR sensor detects the spectral strength in voltage. Fig. 12 shows the inside of designed device.

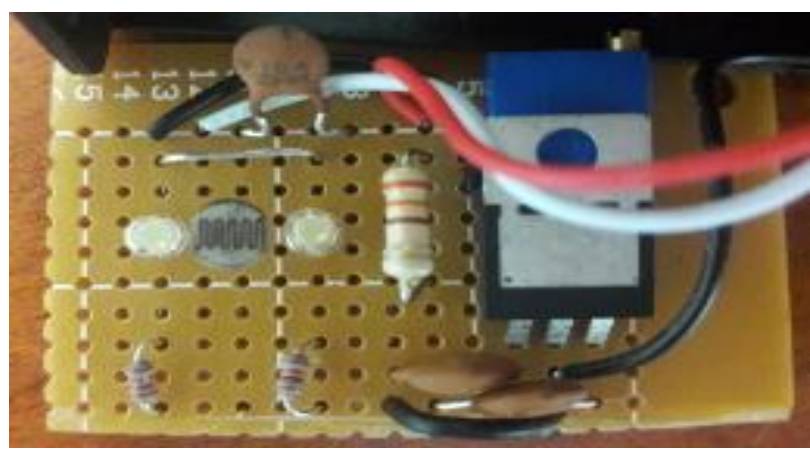

Fig. 12. The inside of the designed sensor device.

Typically, LEDs wavelength range with the single-element output power of at least $5 \mathrm{~mW}$ is 360 to $950 \mathrm{~nm}$. Broadspectrum LEDs light generally are preferred in the reflective production as they are enabled to measure power conceptions. Despite using LEDs, estimates indicate that energy costs for electric lighting can be as high as $25 \%$ to $30 \%$ of operational costs that significantly reduces profits [6]. To measure spectral quality of power consumption, broadband wavelengths are calculated by adding intensities of individual wavelengths within each range. The total intensity between 400-700 nm (IPAR, $\mu \mathrm{mol} \cdot \mathrm{m}^{-2} \cdot \mathrm{s}^{-1}$ ), 400-499 $\mathrm{nm}$ (Iblue, $\mu \mathrm{mol} \cdot \mathrm{m}^{-2} \cdot \mathrm{s}^{-1}$ ) $500-599 \mathrm{~nm}$ (Igreen, $\mu \mathrm{mol} \cdot \mathrm{m}^{-2} \cdot \mathrm{s}^{-1}$ ) and 600 $700 \mathrm{~nm}$ (Ired, $\mu \mathrm{mol} \cdot \mathrm{m}^{-2} \cdot \mathrm{s}^{-1}$ ) broadband wavelength is calculated by adding intensities of individual wavelengths within each range. The percentage of blue, green, and red light output is calculated as the following:

$$
\begin{aligned}
& \text { Blue }=100 * \frac{I_{\text {blue }}}{I_{P A R}}\left(\frac{\mu \mathrm{mol} \cdot \mathrm{m}^{-2} \cdot \mathrm{s}^{-1}}{\mu \mathrm{mol} \cdot \mathrm{m}^{-2} \cdot \mathrm{s}^{-1}}\right) \\
& \text { Green }=100 * \frac{I_{\text {green }}}{I_{P A R}}\left(\frac{\mu \mathrm{mol} \cdot \mathrm{m}^{-2} \cdot \mathrm{s}^{-1}}{\mu \mathrm{mol} \cdot \mathrm{m}^{-2} \cdot \mathrm{s}^{-1}}\right) \\
& \text { Red }=100 * \frac{I_{\text {red }}}{I_{P A R}}\left(\frac{\mu \mathrm{mol} \cdot \mathrm{m}^{-2} \cdot \mathrm{s}^{-1}}{\mu \mathrm{mol} \cdot \mathrm{m}^{-2} \cdot \mathrm{s}^{-1}}\right)
\end{aligned}
$$

In this analysis section, the measurement intensity of $I_{P A R}$, $\mathrm{I}_{\mathrm{red}}, \mathrm{I}_{\mathrm{green}}, \mathrm{I}_{\mathrm{blue}}$ has converted to voltages since the circuit use $24 \mathrm{~V}$ DC. The analog input conversion for PLC has been calculated by the following formula in (4) and (5).

$$
E U=\frac{((\text { EUSpan } *(D A T A-D a t a O f f \operatorname{set}))}{\text { DataSpan }}+\text { EUOffset }
$$


ProcessValue $=\frac{E U}{\text { Factor }}$

where

DATA $=$ Actual data reading in input register.

Factor $=$ arbitrary factor (multiple of 10) needed to get proper resolution and accuracy.

DataSPan $=($ Data at $20 \mathrm{~mA}-$ Data at $4 \mathrm{~mA}) *$ Factor.

$\mathrm{EU}=$ Engineering Units $*$ Factor.

EUOffset $=$ Engineering Units at $4 \mathrm{~mA} *$ Factor .

EUSpan $=($ Engineering Units at $20 \mathrm{~mA}-$ Engineering Units at $4 \mathrm{~mA}) *$ Factor.

DataOffset $=$ Data in input register at $4 \mathrm{~mA}$ (or zero analog signal of whatever range).

Since DC voltage resolution is 0.001 volts, the factor can be chosen as 1000 . The data offset is $3.045 \mathrm{~V}$ without the reflected light due to $24 \mathrm{~V}$ DC power supply. Two LEDs distance is $1 / 2$ inch. RGB color codes are \#CC3300, \#009900 and\#0000CC. According to the reflected light distance of Fig. 11, Table 1 shows the collected data with the difference distance of the reflected color light. In addition, Fig. 13 and Fig. 14 shows the difference distance of the reflected color light.

\begin{tabular}{ccccc}
\multicolumn{4}{c}{ TABLE 1: REFLECTED LIGHT IN DIFFERENCE DISTANCE } \\
\hline $\begin{array}{c}\text { Reflected Light } \\
\text { Distance }\end{array}$ & No & Blue & Green & Red \\
Deflected & Color & Color & Color \\
\hline $\mathrm{D}=1 \mathrm{~mm}$ & $3.05(\mathrm{v})$ & $3.282(\mathrm{v})$ & $3.061(\mathrm{v})$ & $3.678(\mathrm{v})$ \\
& $3.039(\mathrm{v})$ & $3.273(\mathrm{v})$ & $3.259(\mathrm{v})$ & $3.867(\mathrm{v})$ \\
\hline
\end{tabular}

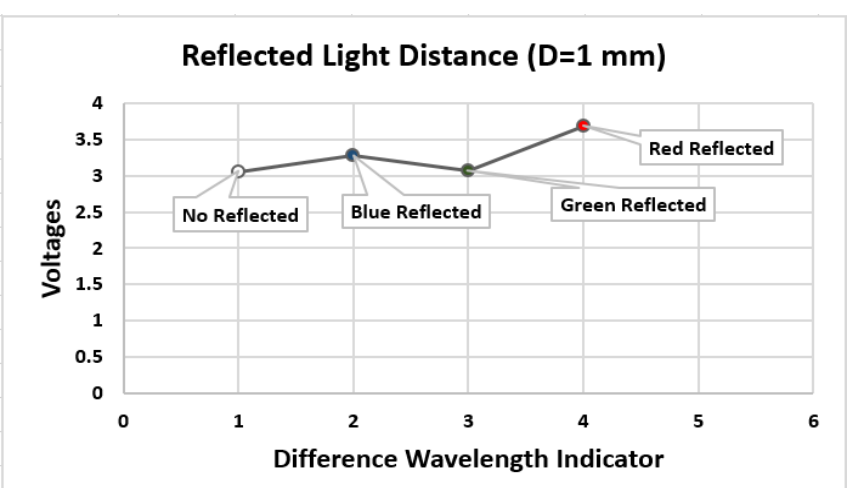

Fig. 13. The reflected light distance $\mathrm{D}=1(\mathrm{~mm})$.

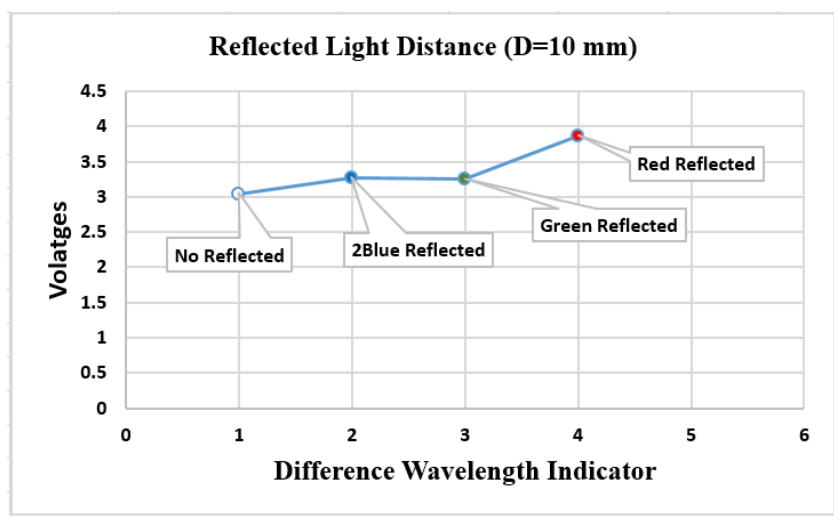

Fig. 14. The reflected light distance $\mathrm{D}=10(\mathrm{~mm})$.
On the other hand, it is important to note that some findings demonstrate that color features like chroma, hue or lightness cause an impact on computing [8]. The hue of RGB color appears to dominate on the reflected light. Their codes are \#FF9999, \#99FF99 and \#66CCFF. In comparison with the difference RGB hue, Table 2 shows the data in the same distance of the reflected light. Fig. 15 shows RGB color hue affects LEDs energy harvest or power consumption.

TABLE 2: The Hue Of BGE Color In The Distance

\begin{tabular}{ccccc}
\multicolumn{5}{c}{$\mathrm{D}=6(\mathrm{MM})$} \\
\hline $\begin{array}{c}\text { Reflected } \\
\text { Light }\end{array}$ & $\begin{array}{c}\text { No } \\
\text { Reflected }\end{array}$ & $\begin{array}{c}\text { Blue } \\
\text { Color }\end{array}$ & $\begin{array}{c}\text { Green } \\
\text { Color }\end{array}$ & Red Color \\
\hline $\begin{array}{c}\text { RGB } \\
\text { (regular) }\end{array}$ & $3.045(\mathrm{v})$ & $3.48(\mathrm{v})$ & $3.375(\mathrm{v})$ & $3.878(\mathrm{v})$ \\
RGB (light) & $3.045(\mathrm{v})$ & $3.71(\mathrm{v})$ & $3.647(\mathrm{v})$ & $4.06(\mathrm{v})$ \\
\hline
\end{tabular}

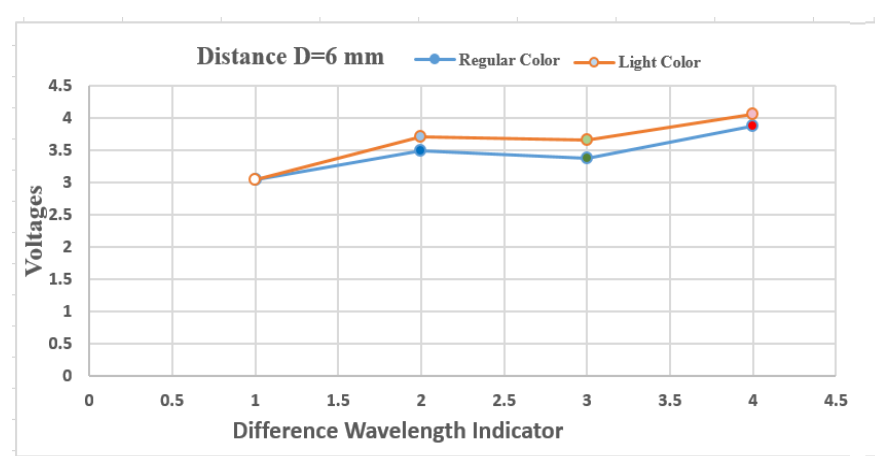

Fig. 15. The comparison of RGB in the difference code.

Moreover, it has proved that the chromatic palette contains the charged information of LEDs energy harvest with respect to the color in particular. The difference in preference between the color codes is obtained for other semantic scales.

\section{CONCLUSIONS}

This paper has introduced a review on the significance of RGB reflected light to color recognition, sorting and counting automation control system. A classification pattern from the color reflected light and the experimental verification of LEDs energy harvest applied to the plastic manufacturing. The distance of the reflected light affected the strength of LEDS energy harvest. Moreover, RGB chroma, hue generally used in affective measuring have been briefly introduced and experiment. The combination of the distance of the reflected light and RGB color is beneficial for the aims of affective measuring in color perception and precision. The concept of methodology and analysis are approved even for the most complex scenarios. Accordingly, the sensor device measured the LEDs energy harvest or power consumption has designed with the sensitive and calibration resistors.

In addition, the designed sensor device measured LEDs energy harvest in voltage had been collected by PLC. HMI interface monitored the quantity of RGB color reflected light and visional analysis by SCADA. Since PLC communicated with SCADA through the IP accessing network, the automation control system can remotely control by internet as 
Internet of Thing (IoT). Furthermore, affective measuring can cause significant improvements in HMI, contributing to enhance the usability of interfaces. Indeed, the automation control system acquires an implicit interaction between humans and machine with the computing facilitates.

\section{REFERENCES}

[1] Design of Colorimeter with RGB Color Sensor, a press release, Agilent Technologies Inc., April 12 $2^{\text {th }}$ 2005, pp.81-90.

[2] Horvat.G, Vinko.D, Švedek.T, LED Powered Identification Tag Energy Harvesting and Power Consumption Optimization, 36th International Convention on Information and Communication Technology, Electronics and Microelectronics, IEEE Xplore, September 2013.

[3] Vullers.R, Schaijk.R, Doms.I, Hoof.C.V, R. Mertens, Micropower Energy Harvesting“, Solid-State Electronics, 5(3), 684-693, 2009.

[4] Frey.L, Parrein.P, Raby.J, Pellé.C, Hérault.D, Marty.M, Michailos.J, Color filters including infrared cut-off integrated on CMOS image sensor, OPTICS EXPRESS, 19(14), 13073-1380, 2011.

[5] Durmus.D, Abdalla.D, Duis.A, Davis.W, Spectral Optimization to Minimize Light Absorbed by Artwork, LEUKOS, 16(1), 45-54, 2020.

[6] Kong.Y, Nemali.A, Mitchell.C, Nemali.K, Spectral Quality of Light Can Affect Energy Consumption and Energy-use Efficiency of Electrical Lighting in Indoor Lettuce Farming, HORTSCIENCE, 54(5), 865-872, 2019.

[7] Tornari.V, Tsiranidou.E, Bernikola.E, Interference fringe-patterns association to defect-types in artwork conservation: an experiment and research validation review, Applied Physics A, 397-410, 2011.

[8] Sokolova.M.V, Fernández-Caballero.A, A Review on the Role of Color and Light in Affective Computing, Applied Sciences, 5(3), 275-293, 2015.

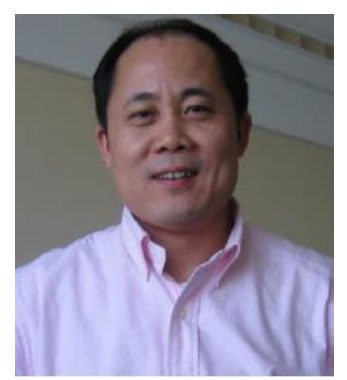

Hong $\mathbf{Y u}$ received the Bachelor degree in Electrical Engineering in 1989, M.S. degree in Electrical Engineering from the Catholic University of America, Washington DC, in 2000 and his Ph.D. degree in Electrical Engineering from the Catholic University of America, Washington DC, in 2008. Currently, $\mathrm{He}$ is an assistant professor of Engineering Technology Department of Fitchburg State University. His teaching and research areas included network communication protocol, embedded system design, VLSI design and simulation, optical switching application, online Laboratory research and renewable energy development.

Alex Pyankov graduated from Fitchburg State University in 2018. He got his Bachelor degree in Electronic Engineering Technology. Currently, he works as senior control system engineer in Boston, USA.

Marcus Perla is a student. He majors on Electronic Engineering Technology at the department of Engineering Technology in Fitchburg State University, USA. 